\title{
Process-Induced Skew Variation for Scaled 2-D and 3-D ICs *
}

\author{
$\mathrm{Hu} \mathrm{Xu}$ \\ LSI-EPFL \\ $\mathrm{CH}-1015$, Switzerland \\ hu.xu@epfl.ch
}

\author{
Vasilis F. Pavlidis \\ LSI-EPFL \\ $\mathrm{CH}-1015$, Switzerland \\ vasileios.pavlidis@epfl.ch
}

\author{
Giovanni De Micheli \\ LSI-EPFL \\ $\mathrm{CH}-1015$, Switzerland \\ giovanni.demicheli@epfl.ch
}

\begin{abstract}
Technology scaling and three-dimensional integration are two design paradigms that offer high device density. Process variations affect these design paradigms in different ways. The effect of process variations on clock skew for a 2-D circuit implemented at scaled technology nodes and for a 3-D circuit with an increasing number of planes is investigated in this paper. An accurate model used to describe the effect of the proper sources of variations on each of these design approaches is proposed. The distribution of the pair-wise skew variation is obtained for single scaled or multi-plane (not scaled) clock distribution networks. The accuracy of the presented statistical skew model is verified through Monte-Carlo simulations. As shown in this paper, the clock skew variation due to technology scaling and/or die stacking exhibits a considerably different behavior. A comparison between these two design paradigms is offered such that the appropriate technology node and number of planes are selected to produce a low clock skew variation and high operating frequency. A popular global clock tree topology is employed in a planar (2-D) circuit where technology scaling is applied and in a 3-D circuit with an increasing number of planes. For this clock tree topology, the maximum supported clock frequency increases from $2.75 \mathrm{GHz}$ to $3.74 \mathrm{GHz}$ by proper die-stacking at a $90 \mathrm{~nm}$ technology node. 3 -D integration is shown to be an alternative to reduce skew variation without the need of aggressive technology scaling.
\end{abstract}

\section{Categories and Subject Descriptors}

B.7.1 [Hardware]: Integrated Circuits_VLSI (very large scale integration)

\section{General Terms}

Performance, Reliability

\footnotetext{
* This work is sponsored by the Swiss National Science Foundation and Intel Germany Labs.
}

Permission to make digital or hard copies of all or part of this work for personal or classroom use is granted without fee provided that copies are not made or distributed for profit or commercial advantage and that copies bear this notice and the full citation on the first page. To copy otherwise, to republish, to post on servers or to redistribute to lists, requires prior specific permission and/or a fee.

SLIP'10, June 13, 2010, Anaheim, California, USA.

Copyright 2010 ACM 978-1-4503-0037-7/10/06 ...\$10.00.

\section{Keywords}

Clock distribution networks, clock skew, technology scaling, process variations, 3-D ICs

\section{INTRODUCTION}

Technology scaling has been the driving force to increase integration density for the past decades. Three-dimensional integration emerges as an alternative design paradigm that can also increase integration density [1]. This increase in density is achieved by plane stacking without necessarily scaling the devices, alleviating problems related to scaling, such as process variations. In very deep sub-micrometer technologies process variations significantly complicate the IC design process $[2,3]$. One of the affected design tasks is the increasing difficulty to produce clock distribution networks with specific performance characteristics. This situation is due to the increase and variation of clock skew, which can limit the maximum operating frequency supported by a clock distribution network.

Clock skew is, typically, defined as the difference between the propagation delays of the clock signal from the source to the sinks of the clock distribution network. High clock frequencies severely constrain clock skew to allow an as large as possible portion of the clock period for data processing. Reducing the interconnect latency can allow relaxed clock skew constraints or a greater circuit speed.

Clock skew is introduced at the design and fabrication stages and during the operation of ICs. There is a plethora of methods to manage the excessive clock skew in the design phase [4-6]. Careful physical design, however, does not guarantee the elimination of undesirable skew since the unwanted skew can be introduced in other phases. Process variability is demonstrated to be an important issue in vertically integrated circuits [7]. The effect of process variations on the performance of 3-D clock distribution networks, however, has not been considered.

The primary sources of process variations include fluctuations of the gate length, doping concentrations, oxide thickness, and interlevel dielectric (ILD) thickness $[2,8]$. The resulting process variations are generally divided to inter-die (die-to-die) and intra-die (within-die) variations. Inter-die variations affect the characteristics of devices independently among dice, but the devices within one die are uniformly affected. Intra-die variations affect the characteristics of devices unequally within one die. Intra-die variations can be characterized as either systematic or random [9-11]. Since both intra- and inter-die process variations are present in a 
3-D IC, the modeling of these process variations is required in the analysis and design of 3-D clock distribution networks.

The effect of intra- and inter-die process variations on the clock skew for scaled 2-D ICs and 3-D ICs with a different number of planes is discussed in this paper. This effect is investigated for a typical global H-tree topology. The proposed model of skew variation can be also used to analyze other 3-D tree topologies generated by clock tree synthesis techniques $[6,12]$.

Results indicate that technology scaling significantly decreases skew variations for clock trees. 3-D integration also decreases skew variation as the number of planes comprising the circuit increases. This reduction, however, is smaller as compared to technology scaling. It is demonstrated that by exploiting both design approaches, the lowest skew variation can be achieved.

The remainder of this paper is organized as follows. Existing techniques for skew analysis in 2-D circuits are briefly reviewed in the following section. A statistical model of the delay of critical paths in 3-D ICs is also discussed. The problem of skew analysis for 3-D clock distribution networks is formulated in Section 3. An accurate clock skew model for 3-D clock trees considering the impact of process variations is described in Section 4. Simulation results and a comparison of 3-D clock trees and scaled 2-D trees are presented in Section 5. The conclusions are drawn in Section 6.

\section{RELATED WORK}

Several techniques for analyzing the effect of process variation on the clock skew have been developed for 2-D circuits emphasizing intra-die variations. Some of these techniques are briefly summarized in this section.

For 2-D ICs, clock skew variations are estimated either by corner analysis or statistical analysis [13]. Corner analysis is useful for estimating inter-die process variations for 2-D ICs but this method often introduces pessimism in the timing of a circuit. Another method for statistical clock skew analysis based on Monte Carlo simulation is introduced in [14]; the computational time of this method is, however, prohibitively high for large scale ICs. Several statistical skew modeling and timing analysis methods considering intra-die variations are presented in $[13,15,16]$ to efficiently analyze skew variations. Although statistical skew analysis has been explored in 2-D ICs, the resulting methods cannot be directly applied to 3-D systems. In 2-D ICs, since the inter-die process variations uniformly affect the devices within a circuit, the majority of the skew analysis methods emphasizes the intradie variations neglecting the impact of inter-die variations.

Recent work analyzing the effect of process variations on the performance of $3-\mathrm{D}$ ICs is presented in $[3,17]$, where the impact of process variations on the delay of critical paths is studied. The distribution of the maximum delay of datapaths is determined by considering that the datapaths have no common segments. Since the clock paths to different sinks can have some common segments, these models are not directly applicable to determine the skew of multiplane clock distribution networks.

The partial correlation of the clock paths and both the inter- and intra-die process variations should be considered in statistical skew analysis for 3-D clock distribution networks. The problem of clock skew modeling under these variations in 3-D ICs is discussed in the following section.

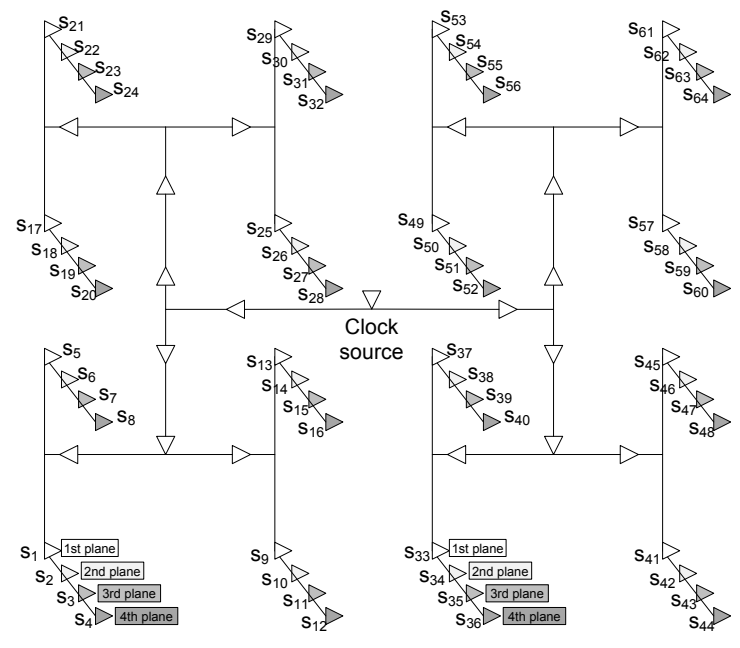

\section{Figure 1: A 3-D H-tree spanning four planes. 3. PROBLEM DESCRIPTION}

The problem of skew analysis for 3-D clock distribution networks considering process variations is described in this section. Since both inter- and intra-die process variations should be considered in 3-D clock distribution networks, the paths starting from the same node cannot be independently treated. Consequently, the previous methods used for determining global skew are not applicable for 3-D clock distribution networks.

Since only the clock skew between the sequential elements which transfer data between each other (data-related sequential elements) affects the performance of a circuit $[5,16]$, in addition to global skew, appropriate pair-wise skew distributions are adopted to evaluate the performance of clock distribution networks [16].

The H-tree is a common topology used to globally distribute the clock signal within a circuit $[4,5]$. A typical buffered 3-D H-tree is illustrated in Fig. $1[1,18]$. The pairwise clock skew used in the following analysis is defined as the skew between every pair of sinks in 3-D clock distribution networks, $M_{\text {skew }}=\left\{m_{i j} \mid m_{i j}=D_{i}-D_{j}, 1 \leq i, j \leq S\right\}$. $m_{i j}$ denotes the skew between sinks $s_{i}$ and $s_{j}$. The clock delay to sinks $s_{i}$ and $s_{j}$ is denoted by $D_{i}$ and $D_{j}$, respectively. The number of clock sinks is $S$.

The number of buffers (determined by the length of interconnects) in clock trees affect the distribution of the delay of clock paths. The area and the number of physical planes constituting a circuit, therefore, significantly affect $m_{i j}$ and the highest supported clock frequency. By investigating the effect of process variations on $M_{\text {skew }}$, the appropriate technology node and number of planes is selected producing a low $M_{\text {skew }}$ and supporting a high clock frequency.

\section{CLOCK SKEW MODELING FOR 3-D CLOCK TREES}

The distribution of clock skew considering inter- and intradie process variations in $3-\mathrm{D}$ clock trees is modeled in this section. The model adopted to obtain the distribution of buffer delay is first presented in Section 4.1. The distribution of the delay of a 3-D clock path and the skew between each pair of sinks in 3-D clock trees is discussed in Section 4.2 and Section 4.3, respectively. 


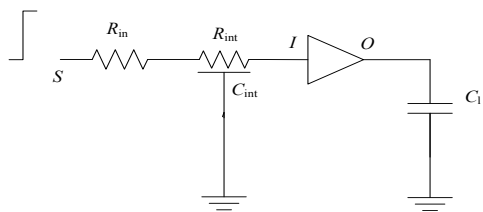

Figure 2: An elemental circuit used to evaluate the variations in the buffer characteristics.

\subsection{Buffer Delay Distribution}

The variation of the buffer delay originates from the device parameters deviating from their nominal values, as statistically modeled in $[8,14]$. The fluctuation of the buffer delay is typically approximated as being linear to the device parameter variations $[16,19]$. Alternatively, the variation in delay can be determined through the variations of the input capacitance and output resistance [13]. The second method is enhanced in this paper to more accurately obtain the buffer delay and the correlation between different buffers. The interconnects constituting the 3-D circuit are modeled as distributed $R C$ wires.

The delay variation of buffers can be estimated by extracting the variations of the device characteristics [8]. Nevertheless, the variation in buffer delay also depends upon the slew rate of the input signal and the load driven by this buffer. The circuit illustrated in Fig. 2 is utilized to obtain the variation of buffer delay for different slew rates of the input signal and $R C$ load. Let $R_{\text {in }}$ denote the output resistance of a buffer. Interconnects with diverse impedance characteristics are modeled by setting different $R_{\text {int }}$ and $C_{\text {int }}$. Different slew rates of the input signal to the buffer in Fig. 2 are, consequently, investigated.

For a step input signal, the Elmore delay [20] variation from source $S$ to nodes $I$ and $O$ in Fig. 2, respectively, is

$$
\begin{aligned}
\Delta D_{S I} & =0.69\left(R_{\text {in }}+R_{\text {int }}\right) \Delta C_{b}, \\
\Delta D_{S O} & =\Delta D_{S I}+\Delta D_{b}+0.69 C_{l} \Delta R_{b},
\end{aligned}
$$

where $\Delta C_{b}, \Delta R_{b}$, and $\Delta D_{b}$ are the variation of the input capacitance, the output resistance, and the intrinsic delay of the buffer, respectively.

Monte Carlo simulations are performed to evaluate the delay variation at nodes $I$ and $O$ where $C_{l}$ is set to two different values (e.g., 0 and $200 \mathrm{fF}$ ). The probability distribution function (PDF) of $\Delta C_{b}, \Delta R_{b}$, and $\Delta D_{b}$ is obtained through (1) and (2). An example of a buffer consisting of two inverters based on a UMC $90 \mathrm{~nm}$ CMOS technology [21] is simulated. The PDF of $\Delta C_{b}, \Delta R_{b}$, and $\Delta D_{b}$ is illustrated in Fig. 3.

The set up of the circuit and the resulting variations of the characteristics of the buffer are reported in Table 1. Slew 1 and slew 2 denote two different slew rates at node $I$ due to two dissimilar pairs of $R_{i n t}$ and $C_{i n t}$. The standard deviation $\sigma$ of the distribution of $\Delta R_{b}, \Delta C_{b}$, and $\Delta D_{b}$ is shown to non-negligibly depend on the input slew. When the slew rate increases, the distribution of $\Delta C_{b}$ and $\Delta D_{b}$ becomes narrower, while the distribution of $\Delta R_{b}$ widens. The statistical delay characteristics of the buffers are, therefore, more accurately captured by considering the different slew rate of the input signal. Since the slew rate and load capacitance of the clock buffers are carefully balanced in well designed

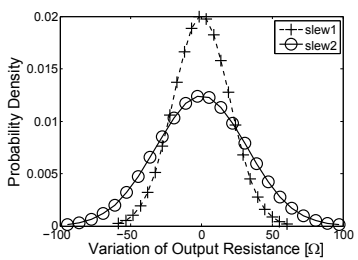

(a)

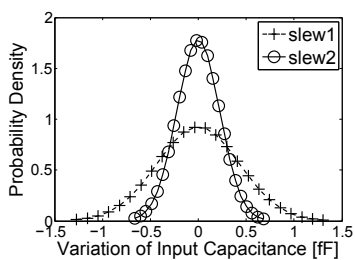

(b)

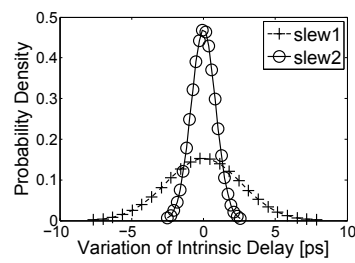

(c)

Figure 3: The PDF of (a) $\Delta R_{b}$, (b) $\Delta C_{b}$, and (c) $\Delta D_{b}$ due to different slew rates of the input signal. The settings of the circuit in Fig. 2 and the resulting variations are listed in Table 1.

clock trees [22], there is a small set of $R_{\text {int }}$ and $C_{i n t}$ which needs to be simulated for a clock tree.

Table 1: Characteristics of the Elemental Circuit and Resulting Variations.

\begin{tabular}{cccc}
\hline Slew rate $[\mathrm{mV} / \mathrm{ps}]$ & $\sigma_{R_{b}}[\Omega]$ & $\sigma_{C_{b}}[\mathrm{fF}]$ & $\sigma_{D_{b}}[\mathrm{ps}]$ \\
\hline 2.57 & 18.33 & 0.43 & 2.45 \\
11.31 & 32.20 & 0.22 & 0.85 \\
\hline
\end{tabular}

The process variations include inter-die (D2D) and intradie (WID) variations, which are independent from each other [10]. $\Delta R_{b}, \Delta C_{b}$, and $\Delta D_{b}$ are each expressed by the summation of the terms produced by D2D and WID variations.

The values of D2D and WID variations can be obtained from experimental data $[10,11]$ or statistical parameters model files [21]. The distribution of D2D and WID variations are modeled as a Gaussian distribution. The mean value and standard deviation are obtained through Monte-Carlo simulations with industrial libraries [21,23].

\subsection{The Delay Distribution of a 3-D Clock Path}

An example of a 3-D clock path is illustrated in Fig. 4. The devices on different physical planes are connected by Through Silicon Vias (TSVs) [24], which, in turn, are modeled as $R C$ wires of different resistance and capacitance as compared to the horizontal wires (e.g., $R_{T S V}$ and $C_{T S V}$ in Fig. 4).

For a clock path consisting of buffers $i-1, i$, and $i+1$, through (1) and (2), the component of the variation of the clock delay $\Delta d_{i}$ related to the delay variation of buffer $i$ but unrelated to the delay variation of other buffers along the same path, is rewritten as

$$
\begin{aligned}
\Delta d_{i} \approx & 0.69\left[R_{i n(i)}^{\prime} \Delta C_{b(i)}+\Delta R_{b(i)} C_{l(i)}^{\prime}\right. \\
& \left.\left.+R_{b(i)}^{\prime} \Delta C_{b(j)}\right)\right]+\Delta D_{b(i)}, \\
R_{i n(i)}= & R_{b(i-1)}+R_{T S V} \\
C_{l(i)}= & 2 C_{i n t}+C_{b(i+1)}+C_{b(j)} .
\end{aligned}
$$




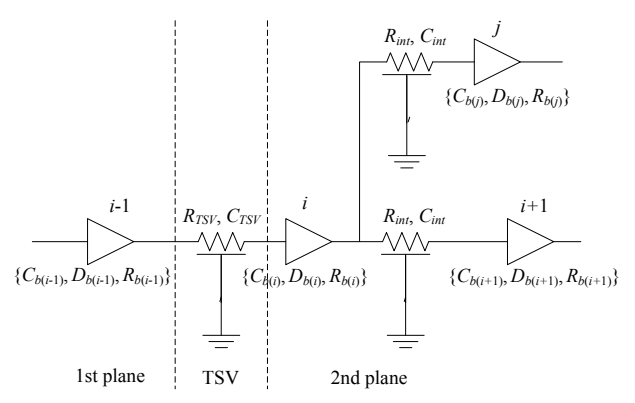

Figure 4: Electrical model of a segment of a clock path.

The prime ( $I$ ) denotes the nominal (mean) values.

The $\Delta R_{b(i)}, \Delta C_{b(i)}$, and $\Delta D_{b(i)}$ are assumed to be fully correlated. According to $(3), \Delta d_{i}$ is approximated as a Gaussian distribution,

$$
f_{\Delta d_{i}}=\mathcal{N}\left(0, \sigma_{d_{i}^{D 2 D}}^{2}+\sigma_{d_{i}^{W I D}}^{2}\right)
$$

Consequently, for a 3-D clock path to a sink $s$ which includes $n_{s}$ clock buffers, the variation of the delay is expressed as the summation of expression (3) applied to each buffer along the path,

$$
\Delta D_{s}=\sum_{i=1}^{n_{s}} \Delta d_{i} .
$$

The WID and D2D sources of $\Delta D_{s}$ are introduced in the following sub-sections, respectively.

\subsubsection{WID Variation Model for the Delay of 3-D Clock Paths}

The correlation of the impact of WID variations on different buffers can be systematic or independent. The systematic WID variations often exhibit spatial correlation [11,25], which can be modeled as a combination of multi-level random WID variations [25]. Consequently, a model for the random WID variations is utilized herein, following the methods used by other statistical skew analysis and process variation analysis papers $[13,15-17]$. The validity of this approach is discussed and demonstrated in these publications. According to (3)-(7), the distribution of the delay of sink $s$ due to WID variations is a Gaussian distribution and the PDF is

$$
f_{\Delta D_{s}^{W I D}}=\mathcal{N}\left(0, \sum_{i=1}^{n_{s}} \sigma_{d_{i}^{W I D}}^{2}\right) .
$$

\subsubsection{D2D Variation Model for the Delay of 3-D Clock Paths}

The variation of the delay of $3-\mathrm{D}$ clock paths due to the $\mathrm{D} 2 \mathrm{D}$ process variations is the sum of the variations of the buffer delay in all the planes,

$$
\Delta D_{s}^{D 2 D}=\sum_{j=1}^{N} \Delta D_{s(j)}^{D 2 D},
$$

where $N$ is the number of the planes that the clock tree spans. $\Delta D_{s(j)}^{D 2 D}$ is the variation of the delay of the clock path from the clock source to sink $s$ in plane $j$. The D2D delay variations of the buffers in the same plane are fully correlated. The distribution of $\Delta D_{s(j)}^{D 2 D}$ is, therefore, a Gaussian

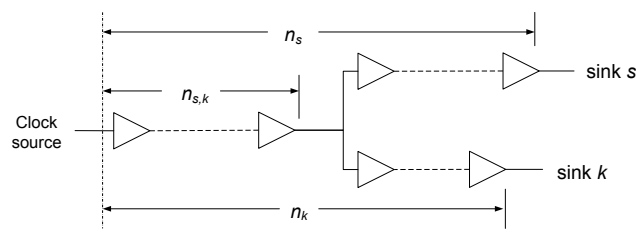

Figure 5: The number of buffers shared by two sinks. distribution and the resulting PDF is

$$
f_{\Delta D_{s(j)}^{D 2 D}}=\mathcal{N}\left(0,\left(\sum_{i=1}^{n_{s(j)}} \sigma_{d_{s(j, i)}^{D 2 D}}\right)^{2}\right),
$$

where $n_{s(j)}$ is the number of buffers located in plane $j$ along this clock path and $d_{s(j, i)}^{D 2 D}$ is the delay related to the $i^{\text {th }}$ buffer in plane $j$.

The D2D variation $\Delta D_{s(j)}^{D 2 D}$ is independent from $\Delta D_{s(k)}^{D 2 D}$ for any $j \neq k$. Consequently, according to (9), the distribution of $\Delta D_{s}^{D 2 D}$ is also a Gaussian distribution and the resulting $\mathrm{PDF}$ is

$$
f_{\Delta D_{s}^{D 2 D}}=\mathcal{N}\left(0, \sum_{j=1}^{N} \sigma_{D_{s(j)}^{D 2 D}}^{2}\right) .
$$

The distribution of $\Delta D_{s}$ is approximated as a Gaussian distribution through (8) and (11).

Some of the above expressions are widely used in modeling process variations. Based on these expressions, a model for the skew variation of $3-\mathrm{D}$ clock trees is proposed in the following section

\subsection{Clock Skew Distribution in 3-D Clock Trees}

For a $3-\mathrm{D}$ clock tree with $S$ sinks distributed in $N$ planes, the skew variation $\Delta m_{s k}$ between sink $s$ and sink $k$ is

$$
\begin{aligned}
\Delta m_{s k} & =\Delta m_{s k}^{W I D}+\Delta m_{s k}^{D 2 D} \\
& =\Delta D_{s}^{W I D}-\Delta D_{k}^{W I D}+\Delta D_{s}^{D 2 D}-\Delta D_{k}^{D 2 D} .
\end{aligned}
$$

According to (8) and (11), the mean value of $\Delta m_{s k}$ is zero. $\Delta D_{s}^{W I D}-\Delta D_{k}^{W I D}$ and $\Delta D_{s}^{D 2 D}-\Delta D_{k}^{D 2 D}$ are independent from each other. Consequently, $\Delta m_{s k}^{W I D}$ and $\Delta m_{s k}^{D 2 D}$ are discussed separately in the following subsections.

\subsubsection{Skew Model of 3-D Clock Trees with WID Vari- ations}

WID variations affect the delay of each buffer independently. According to (8), the distribution of $\Delta m_{s k}^{W I D}=$ $\Delta D_{s}^{W I D}-\Delta D_{k}^{W I D}$ is also a Gaussian distribution,

$$
\begin{aligned}
f_{m_{s k}^{W I D}} & =\mathcal{N}\left(0, \sigma_{m_{s k}^{W I D}}^{2}\right), \\
\sigma_{m_{s k}^{W I D}}^{2} & =\sum_{i=n_{s, k}+1}^{n_{s}} \sigma_{d_{s(i)}^{W I D}}^{2}+\sum_{j=n_{s, k}+1}^{n_{k}} \sigma_{d_{k(j)}^{W I D}}^{2},
\end{aligned}
$$

where $n_{s, k}$ is the number of the buffers shared by the clock paths ending at sinks $s$ and $k$, respectively, as depicted in Fig. 5 .

\subsubsection{Skew Model of 3-D Clock Trees with D2D Vari- ations}

Unlike WID variations, the skew fluctuation caused by $\mathrm{D} 2 \mathrm{D}$ variations is not obtained by adding independent random variables. The correlation between every two terms in the expression of $\Delta m_{s k}^{D 2 D}$ can be one or zero (i.e., fully correlated or uncorrelated, respectively). The skew variation 
$\Delta m_{s k}^{D 2 D}$ can be written as the sum of the terms in different planes,

$$
\begin{aligned}
\Delta m_{s k}^{D 2 D} & =\sum_{j=1}^{N} \Delta m_{s k(j)}^{D 2 D}, \\
\Delta m_{s k(j)}^{D 2 D} & =\sum_{i=1}^{n_{s(j)}} \Delta d_{s(j, i)}^{D 2 D}-\sum_{i=1}^{n_{k(j)}} \Delta d_{k(j, i)}^{D 2 D},
\end{aligned}
$$

where $\Delta d_{s(j, i)}^{D 2 D}$ is the $\mathrm{D} 2 \mathrm{D}$ delay variation related to the $i^{t h}$ buffer on the $j^{\text {th }}$ plane along the clock path ending at sink $s$. The number of buffers in $j^{\text {th }}$ plane along this path is denoted as $n_{s(j)}$.

In (16), all the random variables are equally affected by the $\mathrm{D} 2 \mathrm{D}$ variations in plane $j$, which means that the correlation between every two variables is one. Since $\Delta d_{s(j, i)}^{D 2 D}$ and $\Delta d_{k(j, i)}^{D 2 D}$ are both modeled as Gaussian distributions, $\Delta m_{s k(j)}^{D 2 D}$ is also a Gaussian distribution. In (15), $\forall j_{1} \neq$ $j_{2}\left(1 \leq j_{1}, j_{2} \leq N\right), \Delta m_{s k\left(j_{1}\right)}^{D 2 D}$ is independent from $\Delta m_{s k\left(j_{2}\right)}^{D 2 D}$. Consequently, $\Delta m_{s k}^{D 2 D}$ is also described by a Gaussian distribution,

$$
\begin{aligned}
f_{\Delta m_{s k}^{D 2 D}} & =\mathcal{N}\left(0, \sigma_{m_{s k}^{D 2 D}}^{2}\right) \\
\sigma_{m_{s k}^{D 2 D}}^{2} & =\sum_{j=1}^{N} \sigma_{m_{s k(j)}^{D 2 D}}^{2}, \\
\sigma_{m_{s k(j)}^{D 2 D}} & =\sum_{i=1}^{n_{s(j)}} \sigma_{d_{s(j, i)}^{D 2 D}}-\sum_{i=1}^{n_{k(j)}} \sigma_{d_{k(j, i)}^{D 2 D}} .
\end{aligned}
$$

According to (13) through (19), the skew variation $\Delta m_{s k}$ between $j$ and $k$ in a 3-D clock tree is modeled as a Gaussian distribution,

$$
f_{\Delta m_{s k}}=\mathcal{N}\left(0, \sigma_{m_{s k}^{W I D}}^{2}+\sigma_{m_{s k}^{D 2 D}}^{2}\right) .
$$

This model is used in the following section to investigate the skew variation in $3-\mathrm{D}$ ICs and scaled 2-D ICs for different number of planes and technology nodes, respectively.

\section{SIMULATION RESULTS}

Based on the analysis results in Section 4.3, a global H-tree topology is investigated highlighting the impact of process variations on the clock skew in scaled $2-\mathrm{D}$ and $3-\mathrm{D}$ circuits. The accuracy of the variation model is verified in Section 5.1. Simulation results for scaled 2-D ICs and 3-D ICs with a different number of planes are presented and compared in Section 5.2. The behavior of the skew variation for these design paradigms is also discussed.

\subsection{Accuracy of the Clock Skew Variation Model for 3-D ICs}

The proposed skew variation model is compared with MonteCarlo simulations in this section. The circuit used for this purpose is an H-tree clock distribution network. This Htree is assumed to be placed in a circuit with area $10 \mathrm{~mm} \times$ $10 \mathrm{~mm}$.

The circuit is assumed to be implemented at a $90 \mathrm{~nm}$ CMOS technology. The parameters of the interconnects are obtained from the PTM $90 \mathrm{~nm}$ interconnect model [26]. The clock buffers consist of two inverters connected in series based on a UMC $90 \mathrm{~nm}$ library [21]. The circuit parameters used in the following sections are listed in Table 2. $r_{\text {int }}$ and
Table 2: Device and Interconnect Parameters of Investigated Circuits.

\begin{tabular}{ccccc}
\hline$r_{\text {int }}[\Omega / \mathrm{mm}]$ & $c_{\text {int }}[\mathrm{fF} / \mathrm{mm}]$ & $R_{b}[\Omega]$ & $C_{b}[\mathrm{fF}]$ & $D_{b}[\mathrm{ps}]$ \\
\hline 244.44 & 225.04 & 741.62 & 15.50 & 26.13 \\
\hline$\varnothing_{t s v}[\mu \mathrm{m}]$ & $l_{t s v}[\mu \mathrm{m}]$ & $p_{t s v}[\mu \mathrm{m}]$ & $R_{t s v}[\Omega]$ & $C_{t s v}[\mathrm{fF}]$ \\
\hline 2 & 20 & 10 & 0.13 & 30 \\
\hline
\end{tabular}

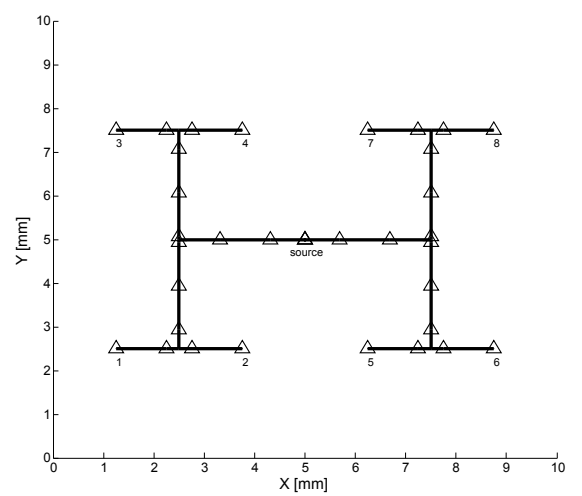

Figure 6: The H-tree used to examine the accuracy of skew variation model.

$c_{\text {int }}$ are the interconnect resistance and capacitance per unit length, respectively. The physical and electrical characteristics of the TSVs are also listed in Table 2 and are based on data reported in [24]. The diameter, length, and pitch of TSVs are, respectively, notated as $\varnothing_{t s v}, l_{t s v}$, and $p_{t s v}$.

The topology of the H-tree used to examine the accuracy of the skew variation model is illustrated in Fig. 6. The clock source is located at the center of the chip. There are eight clock sinks, labeled from one to eight. The clock buffers, which are marked with $\triangle$, are inserted following the technique described in [22]. The wire segments between two buffers are simulated using a standard $\pi$ model. Cadence Spectre is used for the Monte-Carlo simulations [23]. The Monte-Carlo simulation is repeated 1000 times.

Skews $m_{1,2}$ and $m_{1,8}$ are measured to illustrate the accuracy of the adopted model. The curves of cumulative distribution functions (CDF) from Spectre and the proposed skew variation model are shown in Fig. 7 .

The difference between the resulting standard deviation $\sigma$ of Spectre simulation and the skew variation model is within $10 \%$ between any pair of sinks in the investigated clock tree.

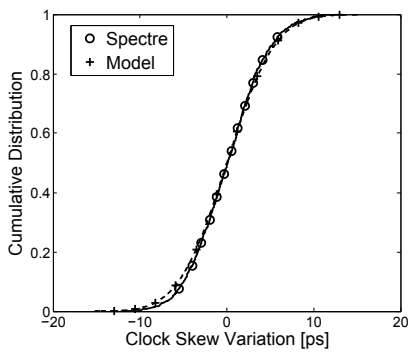

(a)

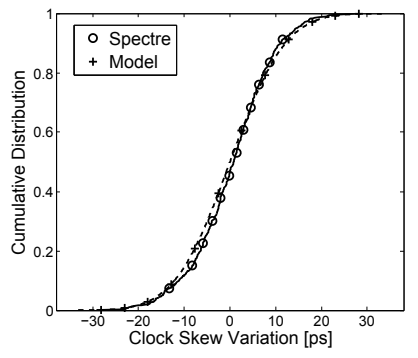

(b)
Figure 7: Comparison of skew variation between Spectre simulations and analytic skew model, where (a) is the CDF of $\Delta m_{1,2}$ and (b) is the CDF of $\Delta m_{1,8}$. 


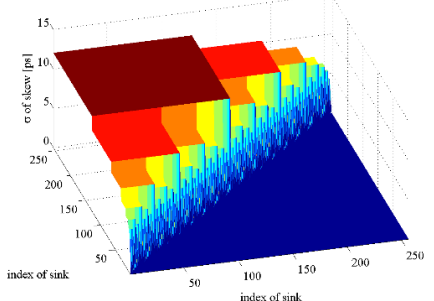

(a)

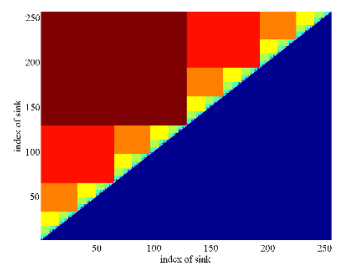

(b)
Figure 8: The $\sigma$ of skew between each pair of clock sinks in a 2-D H-tree. (b) is the top view of (a).

As shown in Figs. 7(a)-7(b), the distribution of the clock skew determined by the skew variation model has a reasonable accuracy as compared with Monte-Carlo simulations.

\subsection{Skew Variation of Scaled 2-D and 3-D Clock Trees}

The skew variation for a typical global $\mathrm{H}$-tree topology in scaled 2-D and 3-D ICs are investigated in this section. The global H-tree topology is shown in Fig. 1. This Htree topology is selected among others $[6,12,27]$ since this topology provides a fairer comparison between the planar and 3-D global clock trees. In this topology, the clock signal is propagated to the sinks in other planes by multiple TSVs.

\subsubsection{Skew Variation of Clock Trees in Scaled 2-D ICs}

For scaled 2-D ICs, since the investigated H-tree topology is symmetric, the number, size, and location of buffers along the paths are equal. The D2D variations, therefore, affect these clock paths equally. Consequently, only WID variations affect the variation of pair-wise skew.

The $\sigma_{m_{i j}}$ between each pair of sinks of a 2-D H-tree is illustrated in Fig. 8. Since $\sigma_{m_{i j}}=\sigma_{m_{j i}}$, only half of the skew matrix is shown in Fig. 8 for clarity. In this example, the electrical parameters are identical to those parameters used in Section 5.1. The area of the circuit is $10 \mathrm{~mm} \times$ $10 \mathrm{~mm}$. There are 256 sinks in the clock tree. A sink can be either a local sub-tree, a clock mesh, or a cluster of registers. $\mathrm{X}$ and $\mathrm{Y}$ axes denote the indices of sinks ordered as described in Fig. 1. As shown in Fig. 8, $\sigma_{m_{i j}}$ changes with the location of sinks $i$ and $j$. The $\sigma_{m_{i j}}$ between the sinks which do not share any segment of a clock path is the largest.

The 2-D H-tree is implemented at $90 \mathrm{~nm}, 65 \mathrm{~nm}, 45 \mathrm{~nm}$, $32 \mathrm{~nm}$, and $22 \mathrm{~nm}$ technology nodes to investigate the effect of process variations on clock skew with technology scaling. The number of sinks is considered to remain invariable throughout these nodes. The area of the circuit is assumed to scale with the feature size of the transistors. The electrical characteristics of the wires and buffers are obtained from ITRS [28]. The resulting largest $\sigma_{m_{i j}}$ of the 2-D clock tree at different technology nodes including process variations is illustrated in Fig. 9, where two variation scenarios are investigated.

In Fig. 9, variation 1 is based on the assumption that $\frac{\sigma_{D_{b}}}{D_{b}^{\prime}}, \frac{\sigma_{R_{b}}}{R_{b}^{\prime}}$, and $\frac{\sigma_{C_{b}}}{C_{b}^{\prime}}$ remain constant for all the technology nodes. Variation 2 is based on the assumptions that $3 \sigma_{L_{e f f}}=10 \% L_{e f f}^{\prime}$ and $3 \sigma_{V_{t h}}=30 \mathrm{mV}$ [26], where $L_{e f f}$ is the effective channel length and $V_{t h}$ is the threshold voltage. As shown in Fig. 9, $\sigma_{m_{i j}}$ decreases with the technology

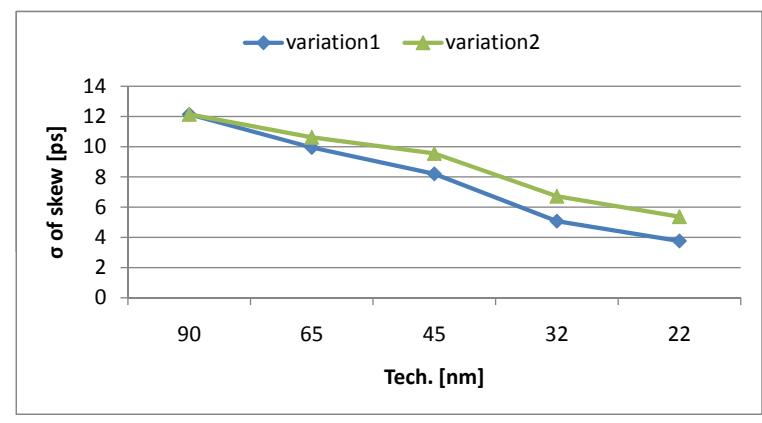

Figure 9: The largest $\sigma$ of skew at different technology nodes.

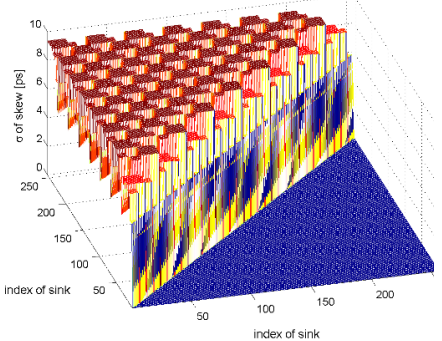

(a)

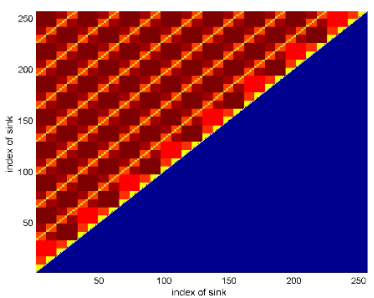

(b)
Figure 10: The $\sigma$ of skew between each pair of clock sinks for a 3-D clock tree topology. (b) is the top view of (a).

scaling for both scenarios. This situation is due to the decrease in $\sigma_{R_{b}}, \sigma_{C_{b}}, \sigma_{D_{b}}$, and the decrease in the circuit area and, consequently, in wire length with technology scaling. The $\sigma_{m_{i j}}$ for variation 2 is larger than variation 1 , since for variation $2, \frac{\sigma_{D_{b}}}{D_{b}^{\prime}}, \frac{\sigma_{R_{b}}}{R_{b}^{\prime}}$, and $\frac{\sigma_{C_{b}}}{C_{b}^{\prime}}$ increase with the decreasing feature size. This situation is due to the effect of process variations which is predicted to increase with technology scaling $[2,28]$.

\subsubsection{Skew Variation in 3-D Clock Trees}

As described by (15)-(19), for 3-D clock trees, the corresponding clock skew is additionally affected by D2D variations. The skew variation between the sinks in different planes vary spatially. The $\sigma_{m_{i j}}$ between each pair of sinks of a 3-D clock tree is illustrated in Fig. 10.

In this example, a 3-D clock tree spanning eight planes is implemented using the topology shown in Fig. 1. The electrical parameters of the wires are given in Section 5.1. There are 32 sinks in each plane and 256 sinks in total. Sinks 1 to 32 are located in the first plane and sinks 33 to 64 are located in the second plane, etc. As an example, consider the $\sigma$ of the skew between sinks 3 and 4 . This variance is determined by the $z$ value of the point $x=3$ and $y=4$. As shown in Fig. 10, $\sigma_{m_{i j}}$ depends on the location of sinks $i$ and $j$. The $\sigma_{m_{i j}}$ between the sinks in the same plane is the smallest.

For 3-D ICs implemented at the same technology node, the clock buffers are inserted by uniform buffer insertion techniques under the same constraints of skew and slew rate. $R_{i n}$ and $C_{l}$ of each buffer, therefore, remain approximately the same. For a $3-\mathrm{D}$ clock tree, as described by (14), the $\sigma_{m_{(s, k)}^{W I D}}$ between two sinks decreases as the number of non- 
Table 3: Mean Value, the Maximum Standard Deviation of Skew, and the Resulting Clock Frequency.

\begin{tabular}{cllllllllll}
\hline \# of planes & 1 & 2 & 3 & 4 & 5 & 6 & 7 & 8 & 9 & 10 \\
\hline$\mu[\mathrm{fs}]$ & 0 & 2.9 & 9.8 & 20.9 & 36.0 & 55.2 & 78.5 & 106 & 137 & 173 \\
$\sigma[\mathrm{ps}]$ & 12.1 & 11.1 & 10.5 & 10.3 & 10.2 & 10.3 & 9.5 & 8.9 & 9.0 & 9.1 \\
$\mu / \sigma[\%]$ & 0 & 0.0 & 0.1 & 0.2 & 0.4 & 0.5 & 0.8 & 1.2 & 1.5 & 1.9 \\
$f_{\max }[\mathrm{GHz}]$ & 2.75 & 3.00 & 3.19 & 3.25 & 3.26 & 3.22 & 3.50 & 3.74 & 3.72 & 3.65 \\
\hline
\end{tabular}

shared clock buffers (e.g., the buffers after the $n_{s, k}$ buffers in Fig. 5) decreases. For 3-D ICs with total area $A_{1}$, the side length of each plane is $L=\sqrt{\frac{A_{1}}{N}}$. The number of buffers in one plane decreases as $L$ decreases for a greater number of planes forming the $3-\mathrm{D}$ circuit.

As the number of planes increases, however, both the number of buffers connected to a TSV and the length of TSVs increase. The load of the buffers driving the TSVs increases, which results in the increase of $\sigma_{d(i)}$, as described by (3). This situation counteracts the benefit of decreasing the number of clock buffers in a plane. Consequently, for the 3-D H-tree, the distribution of pair-wise skew changes non-monotonically as the number of planes increases.

The maximum skew variation of $3-\mathrm{D}$ clock trees spanning different planes is reported in Table 3 . The nominal value $(\mu)$ and the standard deviation $(\sigma)$ of the maximum skew between the first and the topmost planes are reported in Table 3. Note that $\mu$ depends upon the clock tree topology. In 2-D H-trees, $\mu$ is equal to zero. In 3 -D H-trees, the TSVs introduce larger mean skew with the increasing number of planes. The variance is determined by process variations. The importance of process variations is described by $\mu / \sigma$. The skew variation is shown to be dominant as $\mu / \sigma<2 \%$.

The maximum supported clock frequency $f_{\max }$ of a circuit is constrained by the skew [5]. Although $f_{\max }$ is typically determined by the critical path delay, the skew criterion is used here to offer a tangible explanation of the effect of process variations on the performance of circuits. The maximum tolerant skew is assumed to be $10 \% \frac{1}{f_{\max }}$ for the simulated 3 -D clock trees. To achieve a timing yield higher than $99 \%$, $f_{\max }$ should be smaller than $10 \% \frac{1}{m_{i j}(3 \sigma)}$, where $m_{i j}(3 \sigma)$ is the skew at the $3 \sigma$ point from the mean value.

The $f_{\max }$ determined by the skew variation is reported in the last row of Table 3 . The reported $f_{\max }$ changes nonmonotonically with the number of planes, from $2.75 \mathrm{GHz}$ to $3.74 \mathrm{GHz}$. Consequently, the maximum supported clock frequency of this 3 -D clock tree can be improved up to $36 \%$ by selecting the proper number of planes. For a specific technology node, increasing the number of planes can decrease the effect of process variations as compared with a planar circuit, which is also indicated in [3].

The skew variation due to technology scaling and $3-\mathrm{D}$ integration is reported in Table 4 and illustrated in Fig. 11. In Table 4, the first column denotes the technology node and the first row denotes the number of planes of a 3-D circuit. As reported in Table 4 and shown in Fig. 11, the skew variation decreases monotonically with technology scaling but non-monotonically with $3-\mathrm{D}$ integration.

From Table 4 and Fig. 11, the skew variation is shown to decrease more with technology scaling as compared to the increase in the number of planes for a 3-D circuit. For example, the skew variation along column two (scaled 2-D ICs) in Table 4, decreases more as compared to the reduction
Table 4: Standard Deviation of Skew in 3-D ICs with Different Number of Planes and with Technology Scaling. All Values are in ps.

\begin{tabular}{cllllllllll}
\hline \multirow{2}{*}{ Tech. node } & \multicolumn{10}{c}{ \# of planes } \\
\cline { 2 - 11 } & 1 & 2 & 3 & 4 & 5 & 6 & 7 & 8 & 9 & 10 \\
\hline $90 \mathrm{~nm}$ & 12.1 & 11.1 & 10.5 & 10.3 & 10.2 & 10.3 & 9.5 & 8.9 & 9.0 & 9.1 \\
$65 \mathrm{~nm}$ & 10.6 & 10.1 & 9.6 & 9.1 & 8.5 & 8.7 & 8.8 & 8.6 & 8.6 & 8.3 \\
$45 \mathrm{~nm}$ & 9.6 & 9.3 & 9.0 & 7.7 & 8.2 & 8.3 & 8.2 & 7.9 & 7.6 & 7.4 \\
$32 \mathrm{~nm}$ & 6.7 & 5.4 & 6.0 & 6.5 & 6.2 & 6.2 & 5.7 & 6.2 & 6.1 & 5.9 \\
$22 \mathrm{~nm}$ & 5.4 & 4.8 & 5.2 & 4.9 & 4.8 & 4.8 & 4.7 & 4.6 & 4.7 & 4.7 \\
\hline
\end{tabular}

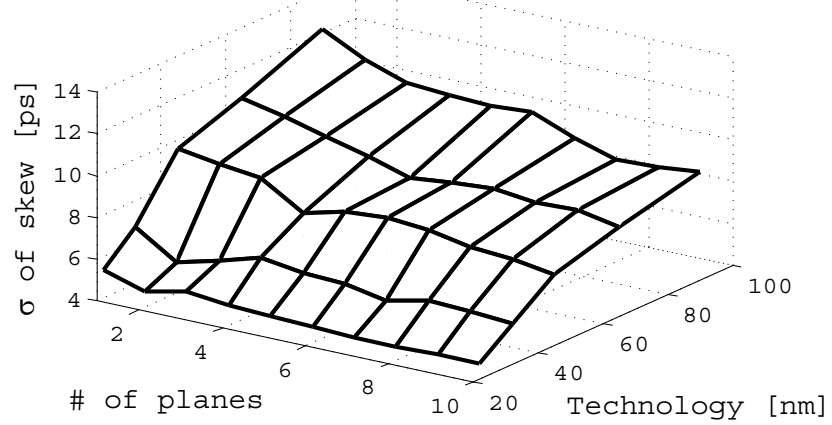

Figure 11: $\sigma$ of skew according to different number of planes at different technology nodes.

in skew variation along the third row (multiplane circuit). Note, however, that the speed of the circuit as determined by the critical path delay increases with the number of planes more as compared with the scaled 2-D circuit as also discussed in $[3,17]$. In addition, a multiplane circuit with a comparable or smaller skew variation can be used instead of a planar 2-D circuit at a smaller technology node. For example, a three plane 3 -D IC at $65 \mathrm{~nm}$ exhibits similar skew variation with a planar circuit at $45 \mathrm{~nm}$ as listed in Table 4. The same applies for a four plane 3 -D IC at $90 \mathrm{~nm}$ and a planar circuit at $65 \mathrm{~nm}$. At deep submicrometer nodes, however, directly increasing the number for a 3-D IC implemented at an older technology node cannot decrease the skew variation as effectively as a deeply scaled planar 2-D circuit.

\section{CONCLUSIONS}

The effect of process variations of devices on the clock skew of scaled 2-D ICs and 3-D ICs is analyzed in this paper. An accurate method to estimate the skew variation within both $2-\mathrm{D}$ and $3-\mathrm{D}$ clock trees considering the effect of the input slew on the clock buffers is presented. Employing the proposed skew model and using an industrial $90 \mathrm{~nm}$ CMOS technology as the baseline technology, skew variation is observed to decrease in different ways between technology scaling and 3-D integration.

A planar global H-tree and an extension of this topology in three dimensions are investigated. Although the skew variation in 2-D ICs with technology scaling decreases more than $3-\mathrm{D}$ integration, for the investigated 3-D clock trees, the resulting maximum supported clock frequency is enhanced up to $36 \%$ by selecting the proper number of planes. $3-\mathrm{D}$ inte- 
gration provides an alternative to offer high device density and low process-induced skew variation without the need of technology scaling. In addition, a combination of technology scaling and vertically integration produces the lowest skew variation in the clock distribution network.

\section{REFERENCES}

[1] V. Pavlidis and E. Friedman, Three-Dimensional Integrated Circuit Design. Morgan Kaufmann, 2009.

[2] S. Nassif, "Delay Variability: Sources, Impacts and Trends," in Proceedings of IEEE International Solid-State Circuits Conference, February 2000, pp. 368-369.

[3] S. Reda, A. Si, and R. Bahar, "Reducing the Leakage and Timing Variability of 2D ICs Using 3D ICs," in Proceedings of IEEE/ACM International Symposium on Low Power Electronics and Design, August 2009, pp. 283-286.

[4] H. B. Bakoglu, J. T.Walker, and J. D. Meindl, "A Symmetric Clock-Distribution Tree and Optimized High-Speed Interconnections for Reduced Clock Skew in ULSI and WSI Circuits," in Proceedings of IEEE International Conference on Computer Design, October 1986, pp. 118-122.

[5] E. Friedman, "Clock Distribution Networks in Synchronous Digital Integrated Circuits," Proceedings of the IEEE, Vol. 89, No. 5, pp. 665-692, May 2001.

[6] T.-Y. Kim and T. Kim, "Clock Tree Embedding for 3D ICs," in Proceedings of Asia and South Pacific Design Automation Conference, January 2010, pp. 486-491.

[7] F. Akopyan et al., "Variability in 3-D Integrated Circuits," in Proceedings of IEEE Custom Integrated Circuits Conference, September 2008, pp. 659-662.

[8] P. Zarkesh-Ha, T. Mule, and J. D. Meindl, "Characterization and Modeling of Clock Skew with Process Variations," in Proceedings of IEEE Custom Integrated Circuits Conference, May 1999, pp. 441-444.

[9] M. Orshansky et al., "Impact of Systematic Spatial Intra-Chip Gate Length Variability on Performance of High-Speed Digital Circuits," in Proceedings of IEEE/ACM International Conference on Computer-Aided Design, November 2000, pp. 62-67.

[10] K. A. Bowman, S. G. Duvall, and J. D. Meindl, "Impact of Die-to-Die and Within-Die Parameter Fluctuations on the Maximum Clock Frequency Distribution for Gigascale Integration," IEEE Journal of Solid-State Circuits, Vol. 37, No. 2, pp. 183-190, February 2002.

[11] K. A. Bowman et al., "Impact of Die-to-Die and Within-Die Parameter Variations on the Clock Frequency and Throughput of Multi-Core Processors," IEEE Transactions on Very Large Scale Integration (VLSI) Systems, Vol. 17, No. 12, pp. 1679-1690, December 2009.

[12] X. Zhao and S. K. Lim, "Power and Slew-aware Clock Network Design for Through-Silicon-Via ( TSV ) based 3D ICs," in Proceedings of Asia and South Pacific Design Automation Conference, January 2010, pp. $175-180$.

[13] X. Jiang and S. Horiguchi, "Statistical Skew Modeling for General Clock Distribution Networks in Presence of Process Variations," IEEE Transactions on Very Large Scale Integration (VLSI) Systems, Vol. 9, No. 5, pp. 704-717, October 2001.

[14] A. Azuma et al., "Methodology of MOSFET Chracteristics Fluctuation Description Using BSIM3v3 SPICE Model for Statistical Circuit Simulations," in Proceedings of International Workshop on Statistical Metrology, June 1998, pp. 14-17.

[15] A. Agarwal, V. Zolotov, and D. T. Blaauw, "Statistical Clock Skew Analysis Considering Intradie-Process Variations," IEEE Transactions on Computer-Aided Design of Integrated Circuits and Systems, Vol. 23, No. 8, pp. 1231-1242, August 2004.

[16] S. Sundareswaran et al., "A Timing Methodology Considering Within-Die Clock Skew Variations," in Proceedings of IEEE International SOC Conference, September 2008, pp. 351-356.

[17] S. Garg and D. Marculescu, "3D-GCP: An Analytical Model for the Impact of Process Variations on the Critical Path Delay Distribution of 3D ICs," in Proceedings of International Symposium on Quality of Electronic Design, March 2009, pp. 147-155.

[18] M. Mondal et al., "Thermally Robust Clocking Schemes for 3D Integrated Circuits," in Proceedings of Conference on Design, Automation and Test in Europe, April 2007, pp. 1206-1211.

[19] A. Devgan and C. Kashyap, "Block-Based Static Timing Analysis with Uncertainty," in Proceedings of IEEE/ACM International Conference on Computer-Aided Design, November 2003, pp. 607-614.

[20] W. Elmore, "The Transient Response of Damped Linear Networks with Particular Regard to Wideband Amplifiers," Journal of Applied Physics, Vol. 19, No. 1, pp. 55-63, January 1948.

[21] Foundry Design Kit (FDK) User Guide, 1.1 ed., United Microelectronics Corporation, August 2007.

[22] G. E. Téllez and M. Sarrafzadeh, "Minimal Buffer Insertion in Clock Trees with Skew and Slew Rate Constraints," IEEE Transactions on Computer-Aided Design of Integrated Circuits and Systems, Vol. 16, No. 4, pp. 333-342, April 1997.

[23] Virtuoso Spectre Circuit Simulator User Guide, 7.0.1 ed., Cadence Design Systems, Inc., June 2008.

[24] G. Katti et al., "Electrical Modeling and Characterization of Through Silicon Via for Three-Dimensional ICs," IEEE Transactions on Electron Devices, Vol. 57, No. 1, pp. 256-262, January 2010.

[25] A. Agarwal, D. Blaauw, and V. Zolotov, "Statistical Timing Analysis for Intra-Die Process Variations with Spatial Correlations," in Proceedings of IEEE/ACM International Conference on Computer-Aided Design, November 2003, pp. 900-907.

[26] "ASU Predictive Technology Model." [Online]. Available: http://www.eas.asu.edu/ ptm/

[27] V. Pavlidis, I. Savidis, and E. Friedman, "Clock Distribution Networks for 3-D Integrated Circuits," in Proceedings of IEEE Custom Integrated Circuits Conference, September 2008, pp. 651-654.

[28] "International Technology Roadmap for Semiconductors," 2008. [Online]. Available: http://www.itrs.net 